\title{
Evidence for reduction of the toroidal ITG instability in the transition from saturated to improved Ohmic confinement in the tokamak TEXTOR
}

\author{
A Kreter, B Schweer, M Z Tokar, B Unterberg and the TEXTOR team \\ Institut für Plasmaphysik, Forschungszentrum Jülich GmbH, EURATOM Association, \\ Trilateral Euregio Cluster, 52425 Jülich, Germany \\ E-mail: a.kreter@fz-juelich.de
}

Received 1 July 2002

Published 31 January 2003

Online at stacks.iop.org/PPCF/45/199

\begin{abstract}
In high density Ohmically heated discharges in the tokamak TEXTOR a transition from the saturated Ohmic confinement (SOC) to the improved Ohmic confinement (IOC) was observed triggered by a sudden reduction of the external gas flow. The SOC-IOC transition was investigated regarding the influence of the toroidal ITG instability driven by the ion temperature gradient (ITG). The ion temperature profiles were measured with high radial resolution by means of charge-exchange recombination spectroscopy (CXRS) with a high-energetic diagnostic hydrogen beam recently installed at TEXTOR. On the basis of the measured ion temperature distributions the $\eta_{\mathrm{i}}$ parameter (ratio of the density and ion temperature decay lengths) and the growth rate of the toroidal ITG instability were calculated. After the SOC-IOC transition $\eta_{\mathrm{i}}$ drops and lies in a noticeably smaller radial region over the threshold for the toroidal ITG. In consequence of it, the IOC regime is characterized by a clear reduction of the ITG growth rate $\gamma_{\text {ITG }}$ which was calculated including finite Larmor radius effects. The steepening of the plasma density profile after the decrease of the external gas flow is the main reason for the reduction of the ITG growth rate and the subsequent confinement transition to the IOC regime.
\end{abstract}

\section{Introduction}

In Ohmically heated discharges the confinement behaviour of the tokamak TEXTOR is characteristic for this type of fusion machines. The occurrence of a confinement regime is directly connected with the plasma density. At low densities the energy confinement time $\tau_{E}$ increases proportionally to the density. This operational mode is called linear Ohmic confinement (LOC). With the density increase the confinement time dependence on density becomes less pronounced and finally saturates. This regime is named saturated Ohmic confinement (SOC). 
In some cases it is possible to overcome the confinement saturation. This regime, where in spite of high density the energy confinement time still scales linearly with that, is called improved Ohmic confinement (IOC) [1]. There are several ways to obtain the IOC regime, e.g. by a slower rise of the plasma density with moderate gas injection [2] or low- $Z$ impurity seeding [3]. In this work, the transition to IOC was achieved by switching off the external gas feed after reaching the confinement saturation in a fast density ramp.

Although these confinement characteristics of Ohmic discharges are well known for a long time, the physical interpretation of the described behaviour is still under discussion. On the one hand, there are numerous theoretical and experimental results [1,3-6], which point to the onset of the toroidal ion temperature gradient (ITG) driven instability $[7,8]$ as the reason for the confinement degradation in the SOC regime. On the other hand, other authors $[9,10]$ deny the crucial role of the ITG instability for the Ohmic confinement.

The fine scale plasma turbulence caused by the ITG instability is widely believed to be the reason for the anomalous transport in tokamaks in discharges with additional heating $[4,11]$. The $\eta_{\mathrm{i}}$ value, which is the ratio of the density and ion temperature decay lengths, is an important parameter governing the ITG instability. On TEXTOR a regime with improved confinement properties was obtained by seeding of the low- $Z$ impurities in discharges with additional heating at densities around the Greenwald limit $n_{\mathrm{GR}}[12,13]$. To describe the mechanisms leading to the transition from the standard L mode to this radiative improved (RI) mode a theoretical model based on the suppression of the toroidal ITG instability was proposed $[14,15]$.

In this paper we investigate the importance of the ITG mode for the SOC-IOC transition. The ion temperature and impurity density profiles were measured with a high radial resolution by means of charge-exchange recombination spectroscopy (CXRS) with a diagnostic hydrogen beam [16] for a series of discharges where the SOC-IOC transition was observed. The experimental results were used to calculate the $\eta_{\mathrm{i}}$ values and the growth rates of the ITG instability $\gamma_{\text {ITG }}$ before and after the transition.

\section{Improved confinement in Ohmic discharges}

The confinement regimes characteristic for TEXTOR [13] are shown in figure 1. As one can see, there are many similarities between discharges with pure Ohmic and additional heating. In fact, the energy confinement time $\tau_{E}$ scales equivalently for LOC/IOC and RI mode with linear dependence on the central line averaged electron density $\bar{n}_{\mathrm{e} 0}[13]$ :

$$
\tau_{E}[\mathrm{~s}]=0.18 \bar{n}_{\mathrm{e} 0}\left[10^{20} \mathrm{~m}^{-3}\right] P_{\mathrm{tot}}^{-2 / 3}[\mathrm{MW}] .
$$

For the SOC and L mode the energy confinement time can be approximated as a function without dependence on the density [13]:

$$
\tau_{E}[\mathrm{~s}]=0.15 I_{\mathrm{P}}[\mathrm{MA}] P_{\mathrm{tot}}^{-2 / 3}[\mathrm{MW}],
$$

where $P_{\text {tot }}$ is the total heating power and $I_{\mathrm{P}}$ is the plasma current. Because of the similarities between the L-RI and SOC-IOC transitions indicated in figure 1 it was obvious to apply the model, which successfully explains the improvement in the RI mode based on the suppression of the toroidal ITG instability $[14,15]$, to the case of the SOC-IOC transition.

A fluid analysis including the finite Larmor radius effect and the polarization drift is used in this paper to calculate the growth rate of the toroidal ITG instability $[15,17]$. For this purpose, the equations of particle, momentum and energy balance for all ion species are linearized with respect to small harmonic perturbations of a frequency $\omega$ and a wave vector $\vec{k}$. The degree of the resulting dispersion relation is three times larger than the number of the ion species including all ionization stages. However, it was found, that besides the main plasma ions only 


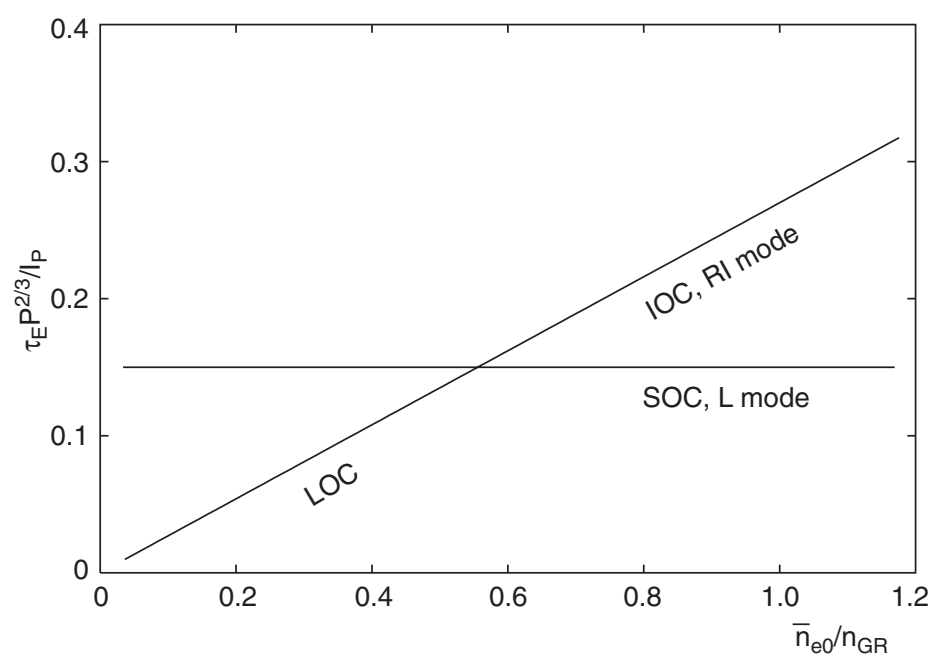

Figure 1. Schematic diagram of the normalized energy confinement time as a function of the normalized density for Ohmic (LOC, SOC and IOC) and additionally heated discharges (L and RI mode) in TEXTOR [13].

fully stripped carbon ions $\mathrm{C}^{6+}$ are of importance in discharges without additional impurity seeding [15]. In discharges with neon seeding, e.g. RI mode, $\mathrm{Ne}^{10+}$ and $\mathrm{Ne}^{8+}$ ions should be also taken into account. The equation of reduced degree can be solved numerically, and the instability growth rate $\gamma_{\text {ITG }} \equiv \operatorname{Im} \omega$ can be calculated.

The factor $\eta_{\mathrm{i}}=L_{n} / L_{T_{\mathrm{i}}}$, where $L_{n}=(-\mathrm{d} \ln n / \mathrm{d} r)^{-1}$ and $L_{T_{\mathrm{i}}}=\left(-\mathrm{d} \ln T_{\mathrm{i}} / \mathrm{d} r\right)^{-1}$ are the decay lengths of the plasma density and ion temperature, respectively, is characteristic for the onset of the mode. To illustrate the effect of profile shapes and impurities on the ITG instability a simplified model with the so-called averaged ion approximation is often used. Thereby the mixture of the main plasma and impurity ions is considered as a single ion sort of charge $Z_{\text {eff }}$. In this approximation an analytical expression can be written for the maximum of the ITG instability growth rate $[14,18]$ :

$$
\gamma_{\mathrm{ITG}} \approx \frac{\omega_{*} \varepsilon_{n}^{1 / 2}}{\tau^{1 / 2} Z_{\mathrm{eff}}^{1 / 2}} \sqrt{\eta_{\mathrm{i}}-\eta_{\mathrm{i}, \mathrm{c}}}
$$

where $\omega_{*}=\left(T_{\mathrm{e}} / e B\right)\left(k_{y} / L_{n}\right)$ is the diamagnetic drift frequency, $k_{y}$ the poloidal wave number, $\tau=T_{\mathrm{e}} / T_{\mathrm{i}}$ and $\varepsilon_{n}=2 L_{n} / L_{\mathrm{B}}$. Thereby we have for the magnetic field scale length $L_{\mathrm{B}}=(-\mathrm{d} \ln B / \mathrm{d} r)^{-1}=R$ in the toroidal geometry. The $\gamma_{\mathrm{ITG}}$ maximum is attained at $k_{y} \approx 0.5 / \rho_{s}$ ( $\rho_{s}$ is the Larmor radius of deuterons) [14]. The value of $\eta_{\mathrm{i}}$ critical for the mode destabilization is:

$$
\eta_{\mathrm{i}, \mathrm{c}} \approx \frac{2}{3}+\varepsilon_{n}\left(\frac{10}{9 \tau Z_{\mathrm{eff}}}+\frac{\tau Z_{\mathrm{eff}}}{4}\right)+\frac{\tau Z_{\mathrm{eff}}}{4 \varepsilon_{n}}-\frac{\tau Z_{\mathrm{eff}}}{2} .
$$

In the RI mode the impurity seeding leads to an initial reduction of the ITG instability growth rate in consequence of the decrease of the diamagnetic drift. The dissipative trapped electron (DTE) instability [19] becomes more important. The DTE mode leads to a density peaking, and due to lower $\eta_{\mathrm{i}}$ the ITG growth rate decreases further. The DTE instability becomes dominating and because of the inverse proportionality of the DTE growth rate to the density $\gamma_{\mathrm{DTE}} \sim 1 / n$ yields the linear dependence of the confinement on the plasma density characteristic for both $\mathrm{RI}$ and IOC regimes. 


\section{Experimental results in discharges with SOC-IOC transition}

To study the SOC-IOC transition at TEXTOR, a medium size tokamak (major radius $R_{0}=1.75 \mathrm{~m}$, minor radius $a=0.46 \mathrm{~m}$ ) of circular cross-section, a series of high density Ohmic discharges at a toroidal magnetic field of $B_{\mathrm{t}}=2.25 \mathrm{~T}$ was performed. Ion and electron temperatures, electron and impurity densities and the diamagnetic energy of the deuterium plasma were measured before and after the transition. The electron density and temperature profiles were measured by the HCN interferometry and ECE diagnostics, respectively.

The ion temperature and impurity density profiles were obtained by means of CXRS with a diagnostic hydrogen beam recently installed at TEXTOR. The injector is based on a rf discharge source developed in the Budker Institute of Nuclear Physics Novosibirsk, Russia, which provides an equivalent neutral current of $1 \mathrm{~A}$ with an extracting voltage of $50 \mathrm{kV}$ [16]. Together with a low angle divergence of $\pm 0.5^{\circ}$ it leads, on the one hand, to a beam current density for sufficiently high charge-exchange signals; on the other hand, the beam heating power and deuterium plasma dilution with hydrogen are negligible, so that measurements in Ohmically heated plasmas are possible. A beam pulse duration of up to $10 \mathrm{~s}$ allows measurements of the ion temperature and impurity densities during the whole TEXTOR discharge with an easy distinction between active CX signal and passive background owing to the modulation of the beam.

Figure 2 shows an Ohmic discharge (plasma current $I_{\mathrm{P}}=400 \mathrm{kA}$, edge safety factor $\left.q_{\mathrm{a}}=3.4\right)$ with a density ramp up to $\bar{n}_{\mathrm{e} 0}=5.5 \times 10^{13} \mathrm{~cm}^{-3}$. At low densities the diamagnetic energy increases proportionally to the plasma density corresponding to the LOC. At high densities there is a saturation of the energy (SOC). The SOC-IOC transition was triggered by a sudden reduction of the external gas flow at the time of $3 \mathrm{~s}$. The diamagnetic energy increases by $10-15 \%$ if we compare it for the corresponding density values before and after the transition. Owing to the fact that at those high densities the ions and electrons are closely coupled (equipartition time between electrons and ions by Coulomb collisions of $\approx 20 \mathrm{~ms}$ ), the confinement improvement is seen in both channels: ions and electrons in the plasma centre become hotter.
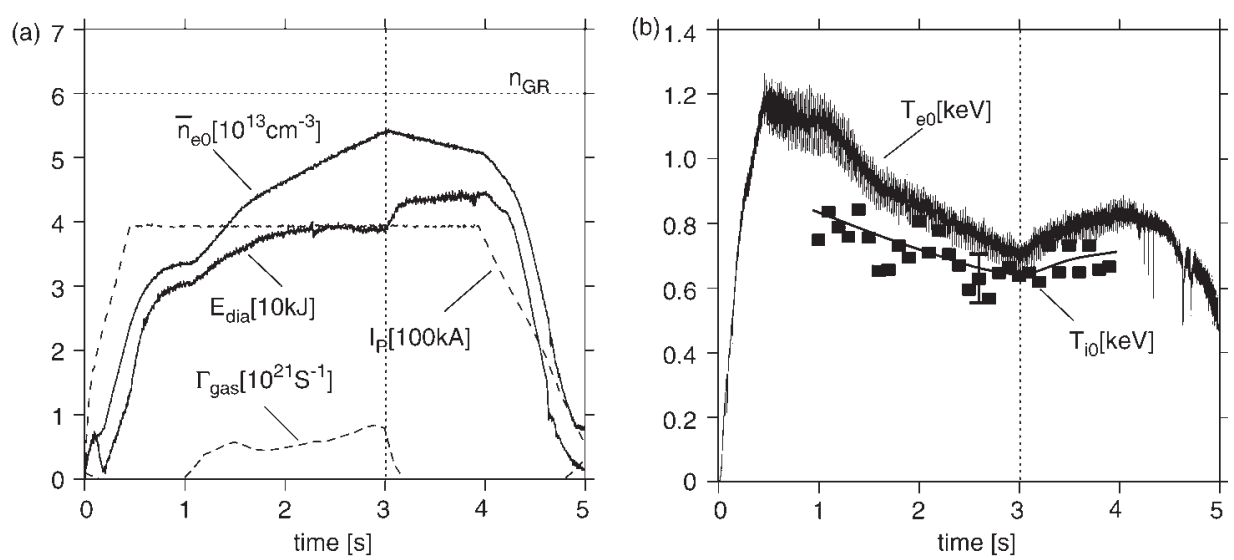

Figure 2. Ohmic discharge (no 85151) with the SOC-IOC transition induced by a sudden reduction of the external gas flow at $3 \mathrm{~s}$ (vertical dotted line). Time traces of: $(a)$ central line averaged electron density $\bar{n}_{\mathrm{e} 0}$ (Greenwald density $n_{\mathrm{GR}}$ is indicated by the horizontal dotted line), diamagnetic energy $E_{\mathrm{dia}}$, plasma current $I_{\mathrm{P}}$ and gas puff flow $\Gamma_{\mathrm{gas}} ;(b)$ central electron and ion temperatures. 


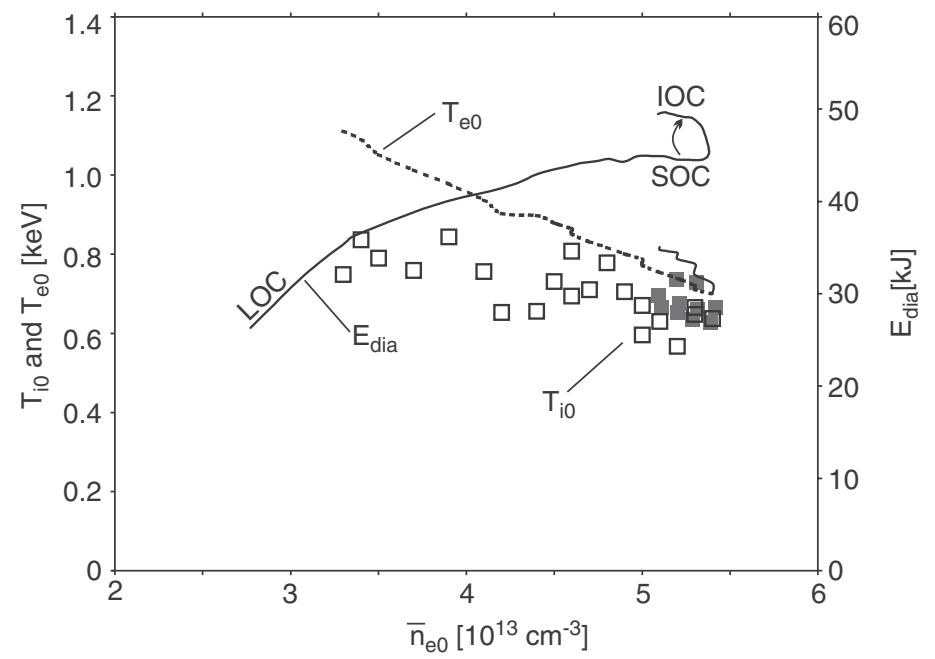

Figure 3. The diamagnetic energy $E_{\text {dia }}$, central ion temperature $T_{\mathrm{i} 0}$ before $(\square)$ and after SOC-IOC transition and electron temperature $T_{\mathrm{e} 0}$ (smoothed over an interval of $100 \mathrm{~ms}$ ) before (- - -) and after (- ${ }_{-}$the transition as functions of the central line averaged electron density $\bar{n}_{\mathrm{e} 0}$ for the flat-top phase of the plasma current (no 85151).

Table 1. Plasma parameters in SOC and IOC (no 87693). $P_{\mathrm{OH}}$ is the Ohmic heating power, $n_{\mathrm{e} 0}$ the central electron density, $n_{\mathrm{i} 0}$ the central ion density, $n_{\mathrm{C}^{6+} 0}$ the central density of $\mathrm{C}^{6+}, Z_{\mathrm{eff}}$ the effective ion charge, $E_{\mathrm{dia}}$ the measured diamagnetic energy, $E_{\mathrm{i}}^{\mathrm{kin}}$ the ion energy derived from the ion temperature and density profiles, $E_{\mathrm{e}}^{\mathrm{kin}}$ the electron energy derived from the electron temperature and density profiles, $\tau_{E}=\left(E_{\mathrm{i}}+E_{\mathrm{e}}\right) / P_{\mathrm{OH}}$ the energy confinement time for stationary conditions.

\begin{tabular}{llllllllll}
\hline & $\begin{array}{l}P_{\mathrm{OH}} \\
\text { Regime }\end{array}$ & $\begin{array}{l}n_{\mathrm{e} 0} \\
(\mathrm{~kW})\end{array}$ & $\begin{array}{l}n_{\mathrm{i} 0} \\
\left(\mathrm{~cm}^{-3}\right)\end{array}$ & $\begin{array}{l}n_{\mathrm{C}^{6+} 0} \\
\left(\mathrm{~cm}^{-3}\right)\end{array}$ & $\begin{array}{l}Z_{\mathrm{eff}} \\
(\mathrm{kJ})\end{array}$ & $\begin{array}{l}E_{\mathrm{dia}} \\
(\mathrm{kJ})\end{array}$ & $\begin{array}{l}E_{\mathrm{e}}^{\mathrm{kin}} \\
(\mathrm{kJ})\end{array}$ & \multicolumn{1}{l}{$\begin{array}{l}\tau_{E} \\
(\mathrm{~ms})\end{array}$} \\
\hline SOC & 380 & $5.9 \times 10^{13}$ & $5.1 \times 10^{13}$ & $1.0 \times 10^{12}$ & 1.5 & 35 & 16 & 20 & 96 \\
IOC & 365 & $6.9 \times 10^{13}$ & $5.8 \times 10^{13}$ & $1.5 \times 10^{12}$ & 1.7 & 41 & 18 & 23 & 112 \\
\hline
\end{tabular}

The diamagnetic energy and the ion and electron temperatures are shown in figure 3 as functions of the plasma density. The evolution of the diamagnetic energy illustrates the confinement regimes of LOC, SOC and IOC described earlier (see figure 1). After switching off of the gas inlet the energy jumps to a higher value at the same density. There is also an increase of the ion and electron temperatures after the transition.

The most important plasma parameters for another discharge with the SOC-IOC transition $\left(I_{\mathrm{P}}=350 \mathrm{kA}, q_{\mathrm{a}}=3.9\right)$ are summarized in table 1 . Regarding the plasma conductivity the rise of the temperature compensates the slight increase of the impurity content in the IOC regime. Therefore there is a weak decrease of the Ohmic heating power. The total plasma energy at those high densities is rather equally distributed between ions and electrons. The energy confinement time rises during the transition approximately by $15 \%$, so that the kinetic energies of both ions and electrons increase.

\section{ITG analysis of the plasma profiles}

Figure 4(a) shows the ion and electron temperature profiles before and after the transition to the IOC. For calculations of $\eta_{\mathrm{i}}, \gamma_{\mathrm{ITG}}$ etc experimental $T_{\mathrm{i}}$ profiles were fitted by analytical 
(a)

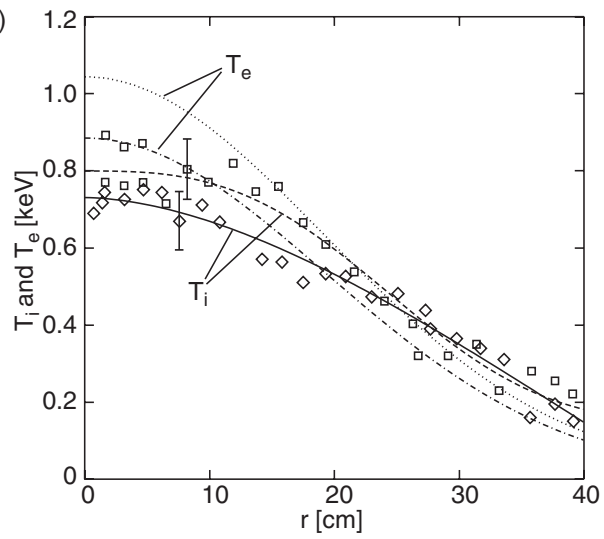

(b)

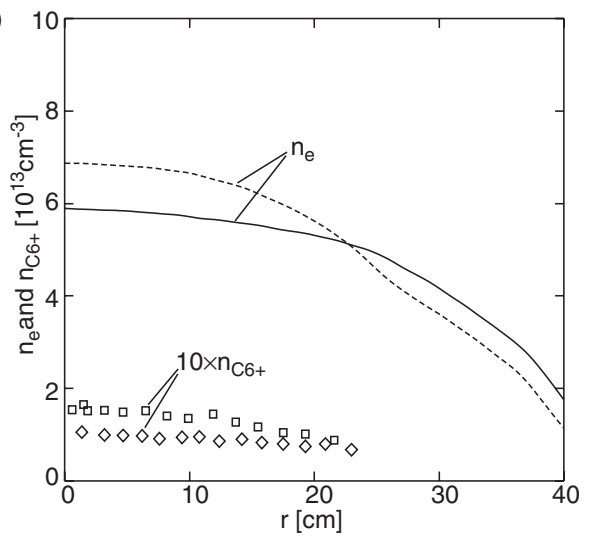

Figure 4. Profiles before and after the SOC-IOC transition (no 87693): (a) the measured ion temperature (SOC: $\diamond$, IOC: $\square$ ), the $T_{\mathrm{i}}$ fit function (SOC: — IOC: - - - ) as well as the electron temperature (SOC: $-\cdots-$ IOC: $\cdots \cdots)$ ); $(b)$ the electron density (SOC: ——, IOC: - - - ) and the density of $\mathrm{C}^{6+}$ (SOC: $\diamond$, IOC: $\square$ ).

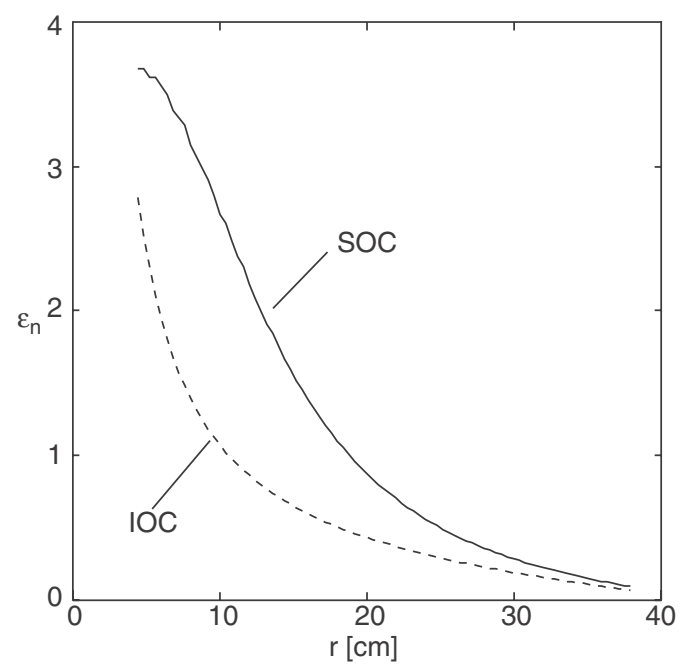

Figure 5. Radial profiles of $\varepsilon_{n}=2 L_{n} / R$ for SOC and IOC (no 87693).

formulae. There is a steepening of the ion and electron temperature profiles as well as of the plasma density profile (figure $4(b)$ ) in the IOC regime. The density of the fully stripped carbon rises after the transition, so that there is an increase of the $\mathrm{C}^{6+}$ concentration in the plasma centre of about $20 \%$. The profiles of $\varepsilon_{n}$, which are directly connected with the shape of the density profile, are shown in figure 5 .

For the profiles shown in figure 4 the $\eta_{\mathrm{i}}$ parameter, which is characteristic for the ITG instability, was calculated. The result is shown in figure 6 along with the profile of its critical value $\eta_{\mathrm{i}, \mathrm{c}}$ (equation (4)). If $\eta_{\mathrm{i}}$ exceeds this critical value, the ITG instability sets on corresponding to the analytical approximation (equation (3)). In the SOC regime $\eta_{\mathrm{i}}$ lies well above $\eta_{\mathrm{i}, \mathrm{c}}$ in a radial region between 7 and $30 \mathrm{~cm}$. That leads to correspondingly high values of the ITG growth rate $\gamma_{\mathrm{ITG}}$ in this region (figure 7). In IOC $\eta_{\mathrm{i}}$ exceeds its critical value 
(a)

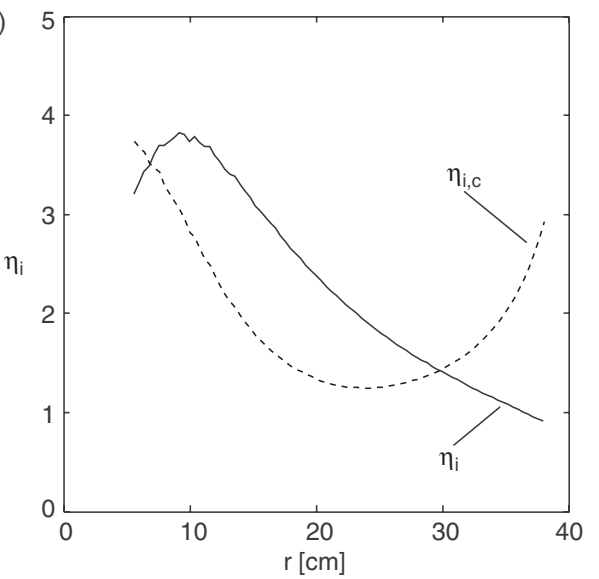

(b)

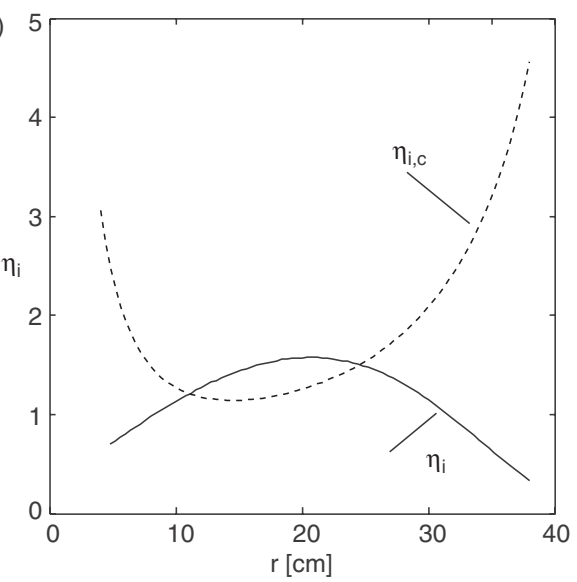

Figure 6. Ratio of the decay lengths of the density and ion temperature $\eta_{\mathrm{i}}$ and its critical value $\eta_{\mathrm{i}, \mathrm{c}}$ (- - - ) in SOC (a) and IOC (b) (no 87693).

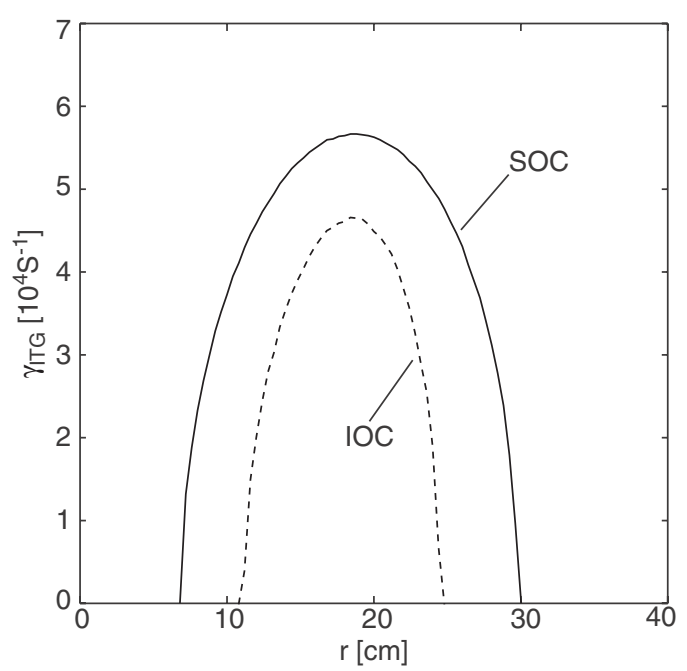

Figure 7. Radial profiles of the ITG instability growth rate (no 87693) calculated using equation (3).

in a significantly narrower region of $11-24 \mathrm{~cm}$. In this region $\gamma_{\mathrm{ITG}}$ for IOC lies well below its value for SOC, because $\eta_{\mathrm{i}}$ in the improved regime only slightly exceeds $\eta_{\mathrm{i}, \mathrm{c}}$.

A more sophisticated analysis was done using an extended model for the ITG instability [15], which includes two ion species, deuterons and carbon nuclei, and regards the finite Larmor radius effects through gyro-viscosity and polarization drift. Figure 8 shows the levels of constant $\gamma_{\text {ITG }}$ computed by this model as a function of the minor radius and the normalized poloidal wave number $k_{y} \rho_{s}$. Although both 'simple' and extended models predict a clear reduction of the ITG growth rate in IOC there is some difference in the results. According to figure 7 the 'simple' averaged ion model, which implies $k_{y} \rho_{s}=0$ computing $\eta_{\mathrm{i}, \mathrm{c}}$, predicts that $\gamma_{\text {ITG }}$ reduces to zero in the outer radial region, where the density profile is steep and $\varepsilon_{n}$, consequently, is small. Conversely, the extended model predicts for this region in the radial 
(a)

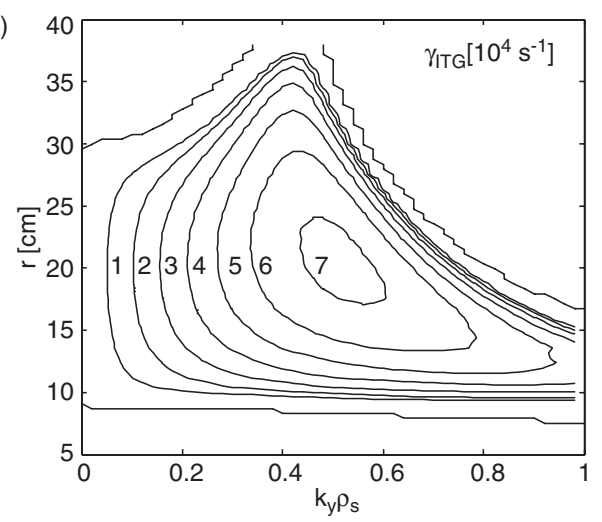

(b)

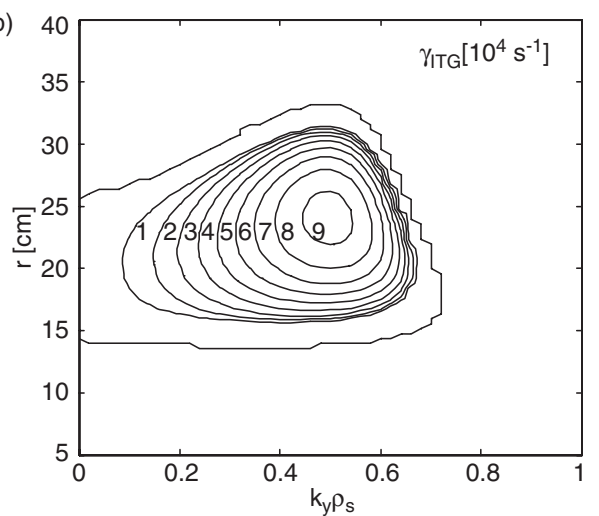

Figure 8. ITG instability growth rate (no 87693) as a function of plasma radius and normalized poloidal wave number for SOC $(a)$ and IOC $(b)$.

zones between 30 and $40 \mathrm{~cm}$ in SOC and 26 and $33 \mathrm{~cm}$ in IOC a gradually narrowing of the growth rate spectrum in the vicinity of $k_{y} \rho_{s} \approx 0.4-0.5$.

The observed reduction of the toroidal ITG instability could be the reason of the confinement improvement in IOC in comparison to SOC as suggested for the case of the L-RI transition in the discharges with additional heating [14]. But unlike the L-RI transition the ITG reduction in Ohmic discharges cannot be triggered by the increase of the impurity concentration. There is only a slight rise of the effective ion charge $Z_{\text {eff }}$ from 1.5 to 1.7 during the SOC-IOC transition, which does not influence the growth of the ITG mode significantly.

Several possible mechanisms are under discussion, how a strong gas puff can cause increased anomalous transport at the edge, e.g. the drift resistive ballooning instability [20,21] or destabilization of the drift waves by the friction of plasma ions with neutrals $[21,22]$. In the SOC regime the external gas inflow can lead to an increase of the edge transport and to a broadening of the plasma density profile resulting in the destabilization of the ITG modes. The reduction of the gas inflow and, connected to it, decrease of the plasma density and neutral pressure at the edge can lead to a reduction of the edge transport resulting in the steepening of the density profile. This steepening leads, as in the case of the L-RI transition, to the reduction of the ITG instability and to the confinement improvement observed in the IOC.

\section{Summary}

High density Ohmically heated discharges in the regimes of the saturated and improvement confinement were investigated in the tokamak TEXTOR. Both stages were compared with respect to the stability of the toroidal ITG mode. For the analysis of the SOC-IOC transition a model was applied, which successfully described the L-RI bifurcation in additionally heated discharges on TEXTOR.

Besides other plasma properties relevant for the model, the ion temperature profiles were measured with a high radial resolution by CXRS with a new diagnostic hydrogen beam. The modification of the profiles in the IOC regime leads to a significant reduction of the growth rate of the toroidal ITG instability. Furthermore, we observe a reduction of the spectral width of the ITG growth rate in a wide radial region. Considering these facts, we can conclude that the toroidal ITG instability, which is responsible for the transport enhancement in the SOC case, is significantly reduced in the IOC regime. 


\section{Acknowledgments}

The authors are very grateful to A A Ivanov, V I Davydenko, P P Deichuli, V I Savkin and the whole diagnostic injector development team of the Budker Institute of Nuclear Physics Novosibirsk, Russia, for providing the principal item of the charge exchange diagnostic, the diagnostic hydrogen beam.

\section{References}

[1] Söldner F X et al 1988 Phys. Rev. Lett. 611105

[2] Sengoku S 1987 J. Nucl. Mater. 145-147 556

[3] Bessenrodt-Weberpals M et al 1991 Nucl. Fusion 31155

[4] Romanelli F, Tang W M and White R B 1986 Nucl. Fusion 261515

[5] Vershkov V A et al 1999 Nucl. Fusion 391775

[6] Rettig C L et al 2001 Phys. Plasmas 82232

[7] Horton W Jr, Choi D-I and Tang W M 1981 Phys. Fluids 241077

[8] Guzdar P N et al 1983 Phys. Fluids 26673

[9] Simmet E E et al 1997 Plasma Phys. Control. Fusion 39993

[10] Becker G 1990 Nucl. Fusion 302285

[11] Dominguez R R and Waltz R E 1987 Nucl. Fusion 2765

[12] Messiaen A M et al 1996 Phys. Rev. Lett. 772487

[13] Weynants R R et al 1999 Nucl. Fusion 391637

[14] Tokar M Z et al 2000 Phys. Rev. Lett. 84895

[15] Tokar M Z et al 1999 Plasma Phys. Control. Fusion 41 L9

[16] Ivanov A A et al 2000 Rev. Sci. Instrum. 713728

[17] Guo S C and Weiland J 1997 Nucl. Fusion 371095

[18] Weiland J 2000 Collective Modes in Inhomogeneous Plasma (Bristol: Institute of Physics Publishing) p 132

[19] Kadomtsev B B and Pogutse O P 1971 Nucl. Fusion 1167

[20] Guzdar P N et al 1993 Phys. Fluids B 53712

[21] Kalupin D et al 2001 Plasma Phys. Control. Fusion 43945

[22] Daughton W et al 1998 Phys. Plasmas 52217 